Research Paper

\title{
The effect of humidified heated breathing circuit on core body temperature in perioperative hypothermia during thyroid surgery
}

Hue Jung Park ${ }^{1}$, Ho Sik Moon², Se Ho Moon³, Hyeon Do Jeong', Young Jae Jeon¹, Keung Do Han5, Hyun Jung Koh1 ${ }^{1 凶}$

1. Department of Anesthesiology and Pain Medicine, College of Medicine, Seoul St. Mary's Hospital, The Catholic University of Korea, Seoul, Republic of Korea

2. Department of Anesthesiology and Pain Medicine, College of Medicine, Yeouido St. Mary's Hospital, The Catholic University of Korea, Seoul, Republic of Korea

3. Department of Anesthesiology and Pain Medicine, College of Medicine, Uijeongbu St. Mary's Hospital, The Catholic University of Korea, Seoul, Republic of Korea

4. Department of Anesthesiology and Pain Medicine, College of Medicine, St. Paul's Hospital, The Catholic University of Korea, Seoul, Republic of Korea

5. Department of Biostatistics, College of Medicine, The Catholic University of Korea, Seoul, Republic of Korea

$\square$ Corresponding author: Hyun Jung Koh, M.D., Ph.D., Department of Anesthesiology and Pain Medicine, College of Medicine, Seoul St. Mary's Hospital, The Catholic University of Korea, 222 Banpo-daero, Seocho-gu, 06591, Seoul, Republic of Korea. Tel: 82-2-2230-6156, Fax: 82-2-537-1951, E-mail: uglyko@hanmail.net

(c) Ivyspring International Publisher. This is an open access article distributed under the terms of the Creative Commons Attribution (CC BY-NC) license (https:// creativecommons.org/licenses/by-nc/4.0/). See http://ivyspring.com/terms for full terms and conditions.

Received: 2017.01.24; Accepted: 2017.05.21; Published: 2017.07.18

\begin{abstract}
Purpose: During general anesthesia, human body easily reaches a hypothermic state, which is mainly caused by heat redistribution. Most studies suggested that humidified heated breathing circuits $(\mathrm{HHBC})$ have little influence on maintenance of the core temperature during early phase of anesthesia. This study was aimed at examining heat preservation effect with HHBC in case of undergoing surgery with less exposure of surgical fields and short surgical duration.

Methods: Patients aged 19 to $70 \mathrm{yr}$ - old, ASA-PS I or II who were scheduled for elective thyroidectomy were assigned and divided to the group using $\mathrm{HHBC}(\mathrm{Gl})$ and the group using conventional circuit (G2) by random allocation. During operation, core, skin, and room temperatures were measured every 5 minutes by specific thermometer.

Results: G1 was decreased by a lesser extent than G2 in core temperature, apparently higher at 30 and 60 minutes after induction. Skin and room temperatures showed no differences between the two groups $(p>0.05)$. Consequently, we confirmed HHBC efficiently prevented a decrease in core temperature during early period in small operation which has difficulty in preparing warming devices or environments were not usually considered.

Conclusions: This study showed that HHBC influences heat redistribution in early period of operation and can lessen the magnitude of the decrease in core body temperature. Therefore, it can be applied efficiently for other active warming devices in mild hypothermia.
\end{abstract}

Key words: Body Temperature, Circuit, Hypothermia, Thyroidectomy

\section{Introduction}

The human body is composed of two compartments for maintaining temperature. One is the central compartment, which is responsible for core temperature. The other is the peripheral compartment, such as musculoskeletal system, which plays a role as the buffer in the thermoregulatory system [1]. Generally, body temperature changes due to circadian rhythm, sex, patient's disease or various environment factors. In general, anesthesia, interthreshold range, which is only $0.2-0.4^{\circ} \mathrm{C}$ in normal body temperature initially, widens up to ten-fold depending on the anesthetic drugs, allowing 
a hypothermic state to occur easily [2]. Therefore, although mild hypothermia, defined on $34-35.9^{\circ} \mathrm{C}$ is a common phenomenon [3] which leads to complications such as surgical wound infection [4]. The further progressed the state of hypothermia, the greater the chance of side effects. In prevention of these complications, many kinds of methods are used, such as a forced air warming blanket, fluid warming devices, heat-pads, or heated humidified breathing circuit (HHBC) intra-operatively in addition to preoperative warming [5].

A decrease in core temperature is apparent when the operation time is longer than two hours and usually the first decrease appears about 30 minutes after starting the operation. Therefore, many investigators consider body temperature measurement to usually not be necessary during Monitored Anesthetic Care or regional anesthesia, minor procedures, or surgeries completed in less than 30 minutes. In addition, the report said that temperature should be monitored at no more than 15minute intervals during all general anesthesia lasting longer than 60 minutes [6]. Previous studies showed that air blanket and warming air were effective in reducing a decrease in core temperature [7]. However, humidified warming circuit did not show any large difference, especially in wide body exposure and operations lasting longer than two hours [8]. We were interested in the core temperature changes in 60- to 120- minute operations (neither too short nor too long), and considered how we should manage it during this period.

In the case of thyroid surgery, hyperthermia is usually overlooked because of its limited surgical conditions; the operation time takes less than two hours, doesn't need a wide skin exposure, and has smaller incision sites than other surgeries. Therefore, we can ignore the changes in body temperature and prepare no warming devices. However, no matter what the condition is like above, it is important to maintain intraoperative normothermia to prevent postoperative inadvertent complications [9]. We studied the core temperature change in thyroid surgery with humidified heated breathing circuit (HHBC). From this study, we aimed to validate the efficacy of HHBC in minor surgery, and to place emphasis on a more practical application of it for preserving core temperature in normothermia.

\section{Methods}

Prospective, single-blind, randomized study was done with the 230 patients, ASA-PS (American Society of Anesthesiologist Physical Status) I or II, scheduled on elective thyroidectomy from August 2010 to June 2011. All of them were benign or malignant neoplasm in thyroid with normal thyroid function and no endocrine abnormality. We excluded the patients with thyroid disease, such as Grave's disease, thyroiditis, toxic nodular goitor, preoperative body temperature above $38^{\circ} \mathrm{C}$, extreme age (under 18 and over 70), infectious disease, emotional abnormality like anxiety, etc. Before starting this study, according to a computer-generated list of random numbers, patients were allocated randomly to one of the two groups. The two groups were: One (G1) used HHBC (Mega Acer Kit, Ace medical, Seoul, South Korea) and the other (G2), control group which used usual conventional breathing circuit (Disposable breathing circuit, King Systems corp.IN, USA) with electrostatic filter (DAR ${ }^{\mathrm{TM}}$, Covidien, MA, USA). In the study period, 230 patients in total were enrolled. We got Informed Consent from each patient preoperatively. This study was approved by the Institutional Review Board of Yeouido St. Mary's Hospital, The Catholic University of Korea (approval number: SC11OIS10265) and registered with Clinical Research Information Service of Korea National Institute of Health (CRIS, identification number: KCT0001459). To assess initial temperatures, core temperature in tympanic membrane was measured just before entering the operating room. We attached a skin temperature probe (Therma-Temp ${ }^{\circledR}$ Probes, 409, Cincinnati Sub Zero Products Inc., Ohio, USA) onto patient's lateral skin-surface of left lower leg. Anesthesia was induced with intravenous thiopental sodium $(5 \mathrm{mg} / \mathrm{kg})$ and succinylcholine $(1 \mathrm{mg} / \mathrm{kg})$. After patients lost self-respiration, we intubated endotracheal tube then inserted 12Fr. esophageal stethoscope (Esophageal Stethoscope, DeRoyal Industries Inc., Powell, TN, USA) for measuring core temperature and its tip was placed on lower third of esophagus where strong heart sounds with breath sounds are detected [10] before removing the laryngoscope from oral cavity. Each group was connected to the designated breathing circuit. We tried to maintain constant room temperature between $20^{\circ} \mathrm{Cand} 22^{\circ} \mathrm{C}$. Room temperature was measured by electric thermometer placed onto the side wall of the operating room. After finishing the anesthetic setting, the patient was covered surgical draps with an exposure of operation site only. At the beginning of operation, we did not use other active warming devices for excluding sources which influence temperature. We estimated core temperature, itself. For severe hypothermia (less than $34^{\circ} \mathrm{C}$ ) during the operation, we planned to apply active warming methods such as forced-air warming blankets and fluid-warming devices for elevating temperature directly. Because we could not rule out the effect of potential sources of interference, these cases were 
excluded. All temperatures (core, skin, room) were measured every 5 minutes until the end of the surgery. Total fresh gas flow was maintained at 3 $\mathrm{L} / \mathrm{min}$ throughout the operation. An initial set temperature of heated circuit inlet was $36.5-37.0^{\circ} \mathrm{C}$ depending on the initial core temperature and the circuit temperature controlled to set point by outlet temperature automatically. Sample size was calculated to detect a $25 \%$ increase in core temperature for the HHBC compared with conventional breathing circuit (2-sided test with $\alpha=0.05, \beta=0.2$, allowing dropout $20 \%$ ).We compared demographic distribution in two groups using paired t-test and McNemar's test by matching patients' age. We could obtain 85 patients in each group. Statistical analysis was performed with the use of SAS software, version 9.2 (SAS institute, Cary, NC, USA). Baseline characteristics conducted by paired t-test and longitudinal changes between groups were tested with repeated measured ANOVA. For correcting continuous variables, we analyzed by Bonferroni's correction to adjust by time sequence. Statistic significant $P$ value was less than 0.05 . The primary outcome of this study was intraoperative core temperature between two groups at each time point. Secondary outcomes were the degree of decrease in core and skin temperature from initial to the end of operation in each group.

Table 1: Demographic data between humidified heating breathing circuit and conventional circuit at induction

\begin{tabular}{llll}
\hline & G1 & G2 & \\
$\mathrm{N}$ & 85 & 85 & P-value* \\
\hline Age & $49.2 \pm 11.7$ & $47.3 \pm 11.9$ & 0.2941 \\
Sex $(\mathrm{M})$ & $22(25.9)$ & $16(18.8)$ & 0.2693 \\
Core Temperature $\left({ }^{\circ} \mathrm{C}\right)$ & $36.4 \pm 0.2$ & $36.4 \pm 0.3$ & 0.0568 \\
Room Temperature $\left({ }^{\circ} \mathrm{C}\right)$ & $20.1 \pm 1.1$ & $19.9 \pm 1.3$ & 0.5917 \\
Skin Temperature $\left({ }^{\circ} \mathrm{C}\right)$ & $30.24 \pm 1.4$ & $30.3 \pm 2.0$ & 0.6648
\end{tabular}

Data are presented as the mean \pm standard deviation.

G1: humidified heating breathing circuit (HHBC); G2: conventional breathing circuit without humidifier and heat (CBC)

*: comparison with every 5 minutes, respectively

$\mathrm{P}<0.05$ was considered statistically significant

\section{Results}

When we started the study, there were 230 patients assessed for eligibility. There were no dropouts from intraoperative severe hypothermia less than $34^{\circ} \mathrm{C}$. 26 patients who were dropped out were due to missing datum after study, incorrect temperature records and preoperative abnormal temperature of over $37.5^{\circ} \mathrm{C}$ or less than $36^{\circ} \mathrm{C}$. All drop-outs (26 patients) were included in control group. Finally, 204 patients (HHBC group (G1); 119 and control group with conventional circuit (G2); 85) were analyzed. In demographic data, we need to match with age when taking into consideration statistical significance. Each group was composed of 85. After matching, demographic datum showed no differences (age; $P=0.2941$, sex; $P=0.2693$ ) (Table 1 ). And, average core, skin and room temperature during study period didn't show the differences between two groups (core T.; $P=0.0568$, skin T.; $P=0.6648$, room T.; $P=0.5917$ ) (Table 1). In the analysis based on total operation time, each thyroidectomy showed variable surgical duration and all of them represented more than 30 minutes. The largest distribution of it was between 60 and 75 minutes and none were over 120 minutes (Figure 1). When we compared at five-minute intervals, there represented no differences in two groups skin and operating room temperature as well as core temperature in all time sequences. Therefore, after the difference at each time point was applied to the analysis every 30-minutes considering previous study [8], the trends of temperature change in Figure 2 seemed to be different in both groups. However, statistical results didn't show any differences, except core temperature. G1 showed lesser decrease in core temperature than G2 $(P=0.0233)$. Particularly, at 30 and 60 minutes showed an apparent smaller decrease in G1 (30 min; $P=0.0001,60 \mathrm{~min} ; P=0.0026$ ) in each time point (Table 2, Figure 2A). However, skin and room temperature still did not show significant differences (skin; $P=0.5293$, room; $P=0.5997$ ) (Figure $2 \mathrm{~B}$ and $2 \mathrm{C}$ ). During the operation, core temperature was above $35^{\circ} \mathrm{C}$ in mild hypothermic category in both groups and mostly less $1.0^{\circ} \mathrm{C}$ decrease than baseline temperature. There were no extreme hypothermic states and adverse events related with hypothermia during operation.

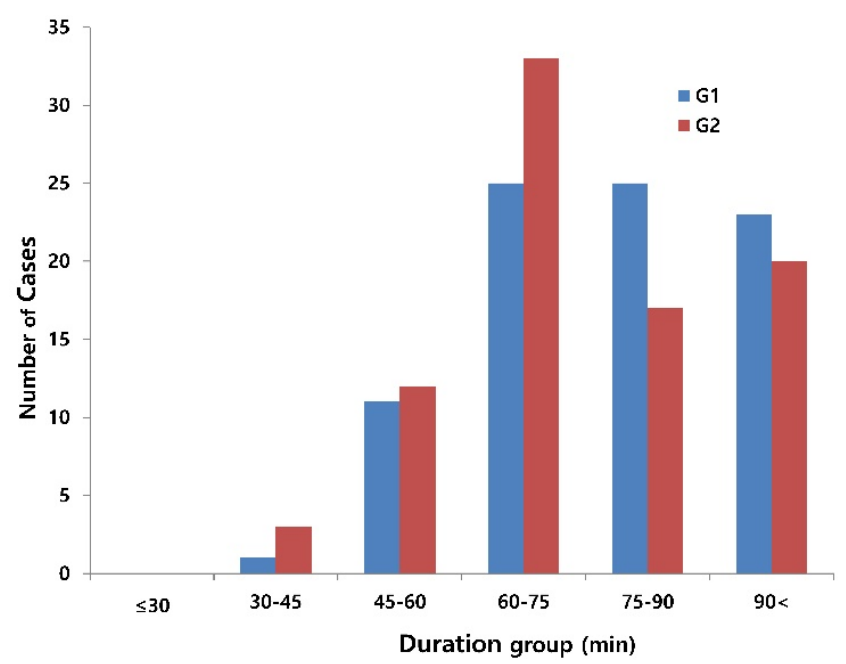

Figure 1. Distribution of total operation time. $x$ : group of total operation time (minutes); $y$ : number of cases (thyroidectomy), red bar (G1): humidified heating breathing circuit (HHBC), blue bar (G2): conventional breathing circuit without humidifier and heat ( $C B C)$ 

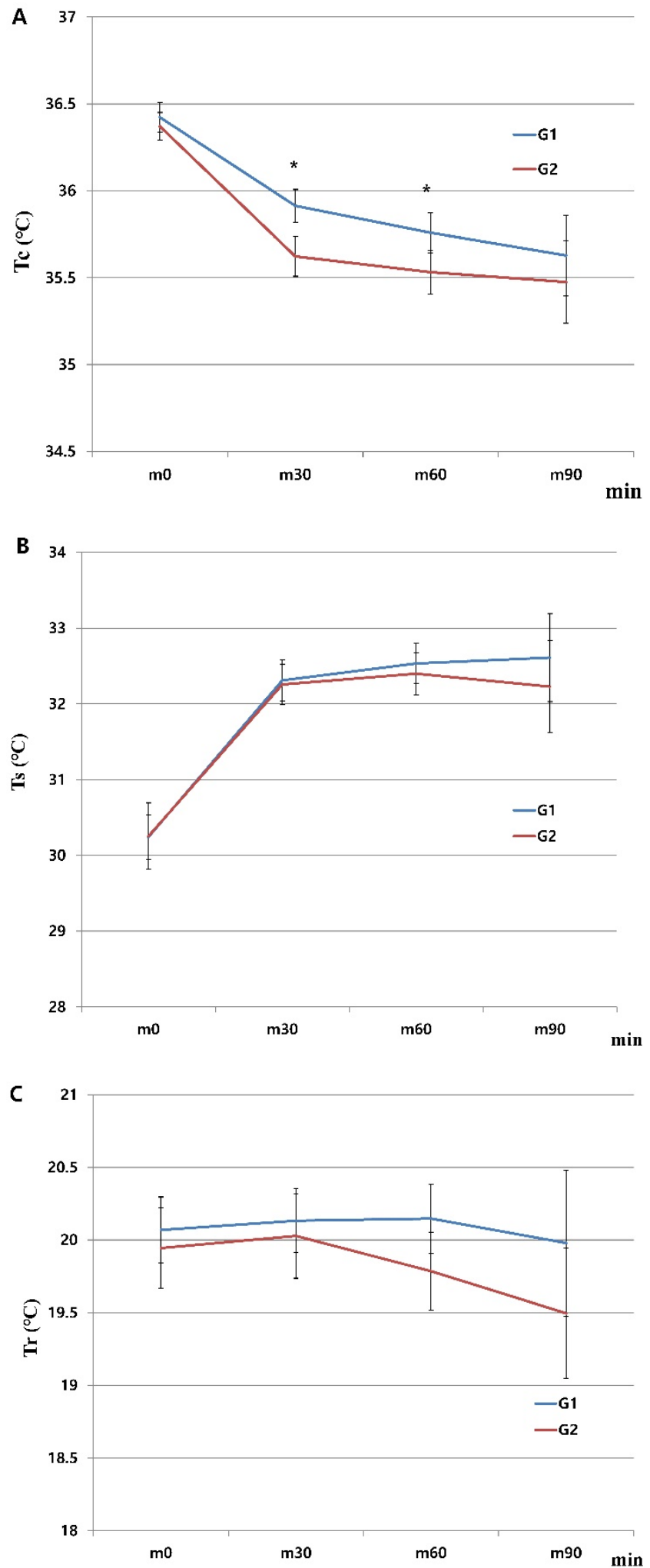

Figure 2. Changes in humidified heating breathing circuit and conventional breathing circuit. A. core temperature; B. skin temperature; C. room temperature. $x$ : minutes; $y$ : ${ }^{\circ} \mathrm{C}$ (Celsius), red line (G1): temperature in humidified heating breathing circuit (HHBC), blue line (G2): temperature in conventional breathing circuit without humidifier and heat (CBC), Tc, core temperature; Ts, skin temperature; $\operatorname{Tr}$, room temperature; $x$, Centigrade $\left({ }^{\circ} \mathrm{C}\right)$; $y$, time (minutes; 30 minutes interval), $* P<0.05$
Table 2: Comparison of initial core temperatures between humidified heating breathing circuit and conventional breathing circuit

\begin{tabular}{llllll}
\hline minutes & $\mathrm{n}$ & $\mathrm{G} 1$ & $\mathrm{n}$ & $\mathrm{G} 2$ & $P$-value \\
\hline Baseline & 85 & $36.42 \pm 0.4$ & 85 & $36.37 \pm 0.37$ & 0.0568 \\
m30 & 85 & $35.91 \pm 0.44$ & 85 & $35.62 \pm 0.53$ & $0.0001^{*}$ \\
m60 & 74 & $35.76 \pm 0.5$ & 70 & $35.53 \pm 0.52$ & $0.0026^{*}$ \\
m90 & 25 & $35.63 \pm 0.56$ & 20 & $35.48 \pm 0.51$ & 0.1586 \\
$P$-value $^{\mathrm{b}}$ & & & & & $0.0233^{*}$
\end{tabular}

Data are presented as the mean \pm the standard deviation.

G1, humidified heating breathing circuit (HHBC); G2, conventional breathing circuit without humidifier and heat (CBC)

G1, G2 horizontal data: temperature $\left({ }^{\circ} \mathrm{C}\right)$

acomparison between $\mathrm{G} 1$ and G2 at 30, 60, and 90 minutes.

bcomparison with 30, 60, and 90 minutes

$* \mathrm{P}<0.05$

\section{Discussion}

Generally, humans try to maintain internal body condition from decreasing body temperature. Under normal conditions, central temperature is maintained within narrow range of $37 \pm 0.2^{\circ} \mathrm{C}$ [11]. There are some risks which will decrease the efficacy of thermoregulatory response and increase in prevalence of hypothermia, such as advanced age, infirmity, medication. Despite circadian rhythm in body temperature, the core temperature is very tightly controlled and regulated by a highly effective system that balances heat production and heat loss. Heat is produced as a consequence of cellular metabolism and lost by radiation, conduction, convection, and evaporation [12].

Core temperature normally ranges from $36.5^{\circ} \mathrm{C}$ to $37.5^{\circ} \mathrm{C}$. The definition of hypothermia is variable. When we look over many reviews, hypothermia is divided into three categories, and the usual concept of hypothermia is less than $36^{\circ} \mathrm{C}$ [13]. Especially perioperative hypothermia is defined as a core temperature of less than $36^{\circ} \mathrm{C}$ [14]. From $35.9^{\circ} \mathrm{C}$ to $34^{\circ} \mathrm{C}$, we call it mild hypothermia [3]. Therefore, it is reasonable to maintain core temperature greater than $36^{\circ} \mathrm{C}$ in surgical patients unless hypothermia is therapeutically indicated [12]. Mostly after surgery, the amount of decrease on core body temperature depends on operation time, range of exposure, operation site, etc. Temperature in the peripheral compartment is usually $2^{\circ} \mathrm{C}$ to $4^{\circ} \mathrm{C}$ lower than the core temperature, and a gradient becomes higher when the anesthesia is induced and vasodilation occurs despite individual differences $[1,14]$.

How to monitor body temperature is a standard of anesthesia care. The continual observation of temperature changes in anesthetized patients allow for the detection of accidental heat loss or malignant hyperthermia [12]. Core temperature can be estimated using probes that can be placed into the distal esophagus, ear canal, trachea and nasopharynx [15]. 
In addition, pulmonary artery blood temperature is also a good indication of core body temperature [12]. But, we selected the esophageal probe because of inaccuracy, inconvenience or invasiveness of other devices [15-18].

During general anesthesia, intraoperative hypothermia is a known consequence [19] and the combination of anesthetic-induced impairment of thermoregulatory control and exposure to a cool operating room environment are the main causes [3]. Core temperature shows a three phase temperature pattern: First, during one hour after the induction, it falls rapidly from 0.5 to $1^{\circ} \mathrm{C}[7,8]$. Second, this is followed by slow, linear decrease. Eventually, it finally plateaus after 2 to 4 hours of anesthesia [1]; this initial rapid onset of hypothermia results from redistribution of heat from the warmer core to the cooler periphery [20].

On the basis of intraoperative core temperature characteristics, we used HHBC to derive the usefulness and proprieties as described below.

In a recent article, they emphasized the fact that more than half the patients had core temperatures below $36^{\circ} \mathrm{C}$ within the first hour of anesthesia. After then, core temperature progressively increased [5]. This means the decrease in core temperature during the first hour results from the particular action (such as heat distribution) rather than external influences mentioned above. In addition, core-to-peripheral redistribution, the main cause of hypothermia during this phase, can remain the dominant cause, even after 3 hours [1]. Therefore, we think that preventing early hypothermia as much as we can is important for lowering adverse outcomes. Thus, if we want to lessen the gap of interthreshold, we have to minimize the core-to-peripheral redistribution. In our study, operation times did not exceed two hours and mostly were less than 90 minutes. Most of them were finished before third phase of temperature drop - between first and second phase of surgical duration. In this condition, the main cause of temperature decline resulted from heat redistribution and a small part from other circumstances [21]. On the basis of this result, we applied HHBC to reduce the decrease in core temperature. However, several studies showed that active airway heating and humidification slightly contribute to the maintenance of central normothermia. Therefore, its efficacy is controversial on either the decreasing of heat loss or the active warming of hypothermia [22-24]. Some studies also state that the main purpose of this warm and humidification is for the optimum level of humidity necessary to prevent drying of secretions and deleterious effects on ciliary function [25]. Consequently, airway heating and humidification are less effective in patients most in need of effective warming [26]. In addition, other reported respiratory heat loss is smaller than radiative heat loss, heating and humidification cannot prevent the temperature drop [7]. Patients undergoing a procedure with general anesthesia lasting longer than 30 minutes are easily exposed to the risk of hypothermia. Therefore, active warming devices such as forced-air warmer, minimum skin exposure and maintenance of optimal room temperature are required [27]. In spite of many kinds of methods, core temperature decreased during first 60-minutes, noticeably after one hour [8]. And forced-air warmer is introduced more effective than circulating water blanket or heated humidifier $[6,28$, 29] in addition to some negative opinions about a heated humidifier in which core temperature became more hypothermic throughout the operation [8]. And, even though it prevents the temperature drop that occurs 30-minutes after induction, it cannot prevent the subsequent drop [30]. In contrast with above results, we controvert these opinions of HHBC. A smaller decrease in core temperature happened apparently between 30 and 60 minutes and it showed a different pattern to existing one, which focused on the last half of initial phase. It suggested that core-to-peripheral temperature change by redistribution can be regulated by HHBC. Even though we cannot confirm how much this circuit influenced heat redistribution, the main cause of initial phase of temperature drop is heat redistribution and we can measure the role of HHBC as a device of temperature modulation. As a result, in consideration of our study, it is improper to ignore use of HHBC at this first phase. In spite of known evidence, HHBC reduced the decrease of core temperature without other devices in our results. The role of HHBC in small operations such as thyroidectomy can be mentioned. Thyroidectomy has some limiting conditions: warming devices cannot be used freely because of narrow operation site, typical surgical position of patients and surgeons, drap coverage pattern and scrub nurse's position. The advantage of application on HHBC is easier access than other thermoregulation devices.

Others suggested that Bair Hugger forced air warming with a surgical access blanket can be used for preventing a decrease in core temperature during anesthesia [31]. However, these forced air warming devices are not cost-effective and in fact difficult to use routinely in our hospital system. Generally, the differences in compensation against the decrease of patient's core temperature depend on preoperative physical status, disease, temperature in operating room, and no innerwear. In this study, we tried to maintain the same conditions for excluding other 
factors; for example, room temperature maintained at $20-22^{\circ} \mathrm{C}$. In most of the surgeries, many conditions can interfere with maintaining the appropriate temperature: difficulty to access the active warming devices in operation field, sudden drop of room temperature by malfunction, low temperature irrigation fluid or main fluid, unopposed wide skin surface exposure, etc. Considering inadvertent conditions like above, we considered the easiest and most effective devices without taking up too much space. The HHBC is suitable for these kinds of surgical conditions. There is another report that intraoperative hypothermia is minimized by 2-hours of active skin-surface warming before starting general anesthesia as well. However, such prolonged pre-warming is impractical in most surgical conditions and hospital systems [32]. From this reason, application of this is also difficult to maintain normal temperature.

When we proceeded with the study, skin and room temperatures had also showed some changes. In particular, there were similar increase patterns until 30 minutes, after then showed slight increase patterns in HHBC groups between 30 to 60 minutes without clinical significances. In any case, this explains that HHBC is advantageous and influences on the peripheral temperature in spite of any statistically proven result. However, further studies are necessary to reveal the dual (core and peripheral temperature) effect of HHBC.

As mentioned above, the conclusions from this study were as follows:

Firstly, HHBC has some advantages: less pace occupied, easy access, and smaller decrease in core temperature, as well as protection of dryness and diluting secretion. Secondly, though Hynson et al. [8] described this humidified heated circuit as useless during any time point of operation, the efficacy of this circuit on protecting a decrease in core temperature is apparent in minor operations which take more than 30-minutes, in contrast to previous suggestions that there is no effect of HHBC compared with other devices [12]. Third, HHBC has an influence on heat redistribution between initial and early second phase temperature drop. Therefore, core temperature can be preserved longer than usual by lowering the effects of vasodilation. From these results, we recommend HHBC for preserving core temperature on early perioperative period or especially in cases lasting less than 60 minutes if other devices are difficult to prepare and have limited conditions. The intension to try this is considered to change the usual concept as a warming device and show positive effect on temperature preservation. In addition, it can be used widely and contribute to the reduction of postoperative complications in unexpected hypothermia.

In this study, there were some limitations to take into consideration.

First is the lack of comparative methods. We did not compare with other active warming devices. We intended to suggest the benefit of HHBS itself. Therefore, further investigation should be tried between humidified heating circuits and other active warming devices. Also, we need to find out whether or not other devices will show similar efficacy compared with heating circuits. Second is range of patient selection. We did not consider the postoperative complications related to hypothermia because of the exclusion of extreme temperature and operative characteristics. In the future, in-depth studies should be done toward the efficacy of HHBC on high-risk patients who are prone to complications. Third is lack of observation period about adverse outcomes related to hypothermia. We observed temperature only in the operating room. Therefore, we could not find any complications related to hypothermia even though we already mentioned the possibility of complications. More specific studies were needed to detect the adverse events such as comparing complications between HHBC and conventional BC. Fourth is no basic temperature conserving method is used in the control arm. Thyroidectomy is included in low-risk, minor surgery in surgical grade classification. As previously mentioned, it has an operation time that is not too long and small incision site which could not influence on heat loss by evaporation. In addition, the recruited patients were involved in ASA-PS class I or II without respiratory or airway problems. After considering these conditions, at first we obtained written informed consent from control group patients of not using HME (Heat and Moisture Exchanger). We just prepared the warming method to manage accidental hypothermia. The last is lack of precision of measurement of core temperature. We used only esophageal stethoscope and low fresh gas flow only 2 L/hr. Heated air can influence esophageal temperature. In consideration of surgical limitations, we have to use esophageal stethoscope. However, we also have to consider applying other measurements for core temperature and further studies should be done about comparison with other measurements for core temperature.

\section{Acknowledgments}

The author thanks the residents who participated in this study for checking and recording the data. Support was provided by Yonggue Park, PhD. professor of the department of Biostatistics in 
College of Medicine, The Catholic University of Korea.

\section{Clinical Trial Number and Registry URL}

We registered in CRIS (Clinical Research Information Service) in Korea.

\section{Competing Interests}

The contents of this study have not been published nor are they being submitted elsewhere. The manuscript has been read and approved by all co-authors. Hue Jung Park, Ho Sik Moon, Se Ho Moon, Hyeon Do Jeong, Young Jae Jeon and Keung Do Han have neither financial disclosures nor conflicts of interests.

\section{References}

1. Sessler DI. Perioperative heat balance. Anesthesiology. 2000; 92: 578-96.

2. Sessler DI. Temperature monitoring and perioperative thermoregulation. Anesthesiology. 2008; 109: 318-38.

3. Kurz A. Thermal care in the perioperative period. Best Pract Res Clin Anaesthesiol. 2008; 22: 39-62.

4. Kurz A, Sessler DI, Lenhardt R. Perioperative normothermia to reduce the incidence of surgical-wound infection and shorten hospitalization. Study of Wound Infection and Temperature Group. N Engl J Med. 1996; 334: 1209-15.

5. Carpenter L, Baysinger CL. Maintaining perioperative normothermia in the patient undergoing cesarean delivery. Obstet Gynecol Surv. 2012; 67: 436-46.

6. Torossian A. Intraoperative temperature management. Anasthesiol Intensivmed Notfallmed Schmerzther. 2008; 43: 397-9.

7. Ouellette RG. Comparison of four intraoperative warming devices. AANA J. 1993; 61: 394-6.

8. Hynson JM, Sessler DI. Intraoperative warming therapies: a comparison of three devices. J Clin Anesth. 1992; 4: 194-9.

9. Kim E, Lee SY, Lim YJ, et al. Effect of a new heated and humidified breathing circuit with a fluid-warming device on intraoperative core temperature: a prospective randomized study. J Anesth. 2015; 29: 499-507.

10. Kaufman RD. Relationship between esophageal temperature gradient and heart and lung sounds heard by esophageal stethoscope. Anesth Analg. 1987; 66: 1046-8.

11. Sessler DI. Thermoregulatory defense mechanisms. Crit Care Med. 2009; 37(7 Suppl): S203-10.

12. Barash PG, Cullen BF, Stoelting RK. Clinical Anesthesia. 5th ed. Philadelphia, USA: Lippincott Williams \& Wilkins; 2006

13. Hart SR, Bordes B, Hart J, Corsino D, Harmon D. Unintended perioperative hypothermia. Ochsner J. 2011; 11: 259-70.

14. Grocott HP, Tran T. Thermoregulation and perioperative hypothermia. In: Longnecker DE, Brown DL, Newman MF, Zapol WM, eds. Anesthesiology, 2nd ed. New York: McGraw-Hill Medical; 2012: 1504-13.

15. Hooper VD, Andrews JO. Accuracy of noninvasive core temperature measurement in acutely ill adults: the state of the science. Biol Res Nurs. 2006; 8: 24-34.

16. Erickson RS, Meyer LT. Accuracy of infrared ear thermometry and other temperature methods in adults. Am J Crit Care. 1994; 3: 40-54

17. Heidenreich T, Giuffre M, Doorley J. Temperature and temperature measurement after induced hypothermia. Nurs Res. 1992; 41: 296-300.

18. Lattavo K, Britt J, Dobal M. Agreement between measures of pulmonary artery and tympanic temperatures. Res Nurs Health. 1995; 18: 365-70.

19. Kadam VR, Moyes D, Moran JL. Relative efficiency of two warming devices during laparoscopic cholecystectomy. Anaesth Intensive Care. 2009; 37: 464-8.

20. Sessler DI. New surgical thermal management guidelines. Lancet. 2009; 374: 1049-50

21. Matsukawa T, Sessler DI, Sessler AM, et al. Heat flow and distribution during induction of general anesthesia. Anesthesiology. 1995; 82: 662-73.

22. Stone DR, Downs JB, Paul WL, Perkins HM. Adult body temperature and heated humidification of anesthetic gases during general anesthesia. Anesth Analg. 1981; 60: 736-41.

23. Tølløfsrud SG, Gundersen Y, Andersen R. Peroperative hypothermia. Acta Anaesthesiol Scand. 1984; 28: 511-5.

24. Bissonnette B, Sessler DI, LaFlamme P. Passive and active inspired gas humidification in infants and children. Anesthesiology. 1989; 71: 350-4

25. Bickler PE, Sessler DI. Efficiency of airway heat and moisture exchangers in anesthetized humans. Anesth Analg. 1990; 71: 415-8.

26. Sessler DI. Perioperative temperature control. West J Med. 1992; 157: 566-7.

27. Hooper VD, Chard R, Clifford T, et al. ASPAN's evidence-based clinical practice guideline for the promotion of perioperative normothermia: second edition. J Perianesth Nurs. 2010; 25: 346-65.
28. Sessler DI Complications and treatment of mild hypothermia. Anesthesiology. 2001; 95: 531-43.

29. Putzu M, Casati A, Berti M, Pagliarini G, Fanelli G. Clinical complications, monitoring and management of perioperative mild hypothermia: anesthesiological features. Acta Biomed. 2007; 78: 163-9.

30. Lee HK, Jang YH, Choi KW, Lee JH. The effect of electrically heated humidifier on the body temperature and blood loss in spinal surgery under general anesthesia. Korean J Anesthesiol. 2011; 61: 112-6.

31. Ihn $\mathrm{CH}$, Joo JD, Chung HS, et al. Comparison of three warming devices for the prevention of core hypothermia and post-anaesthesia shivering. J Int Med Res. 2008; 36: 923-31.

32. Vanni SM, Braz JR, Modolo NS, Amorim RB, Rodrigues GR Jr. Preoperative combined with intraoperative skin-surface warming avoids hypothermia caused by general anesthesia and surgery. J Clin Anesth. 2003; 15: 119-25. 